\title{
A concise enantioselective synthesis of the guaiane sesquiterpene (-)-oxyphyllol
}

\author{
Martin Zahel and Peter Metz
}

\section{Full Research Paper}

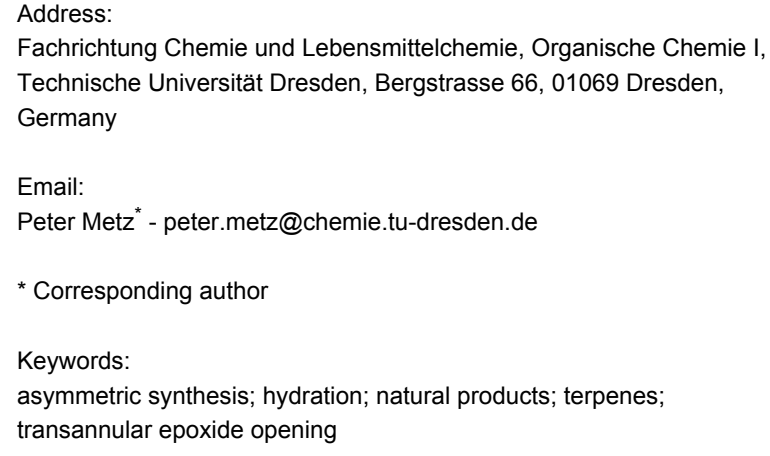

Beilstein J. Org. Chem. 2013, 9, 2028-2032.

doi:10.3762/bjoc. 9.239

Received: 05 August 2013

Accepted: 20 September 2013

Published: 08 October 2013

This article is part of the Thematic Series "Natural products in synthesis and biosynthesis".

Guest Editor: J. S. Dickschat

(c) 2013 Zahel and Metz; licensee Beilstein-Institut.

License and terms: see end of document.

\begin{abstract}
(-)-Oxyphyllol was prepared in only 4 steps from an epoxy enone that already served as an intermediate for the total synthesis of the anticancer guaiane (-)-englerin A. A regio- and diastereoselective $\mathrm{Co}(\mathrm{II})$-catalyzed hydration of the olefin and a transannular epoxide opening were used as the key reactions.
\end{abstract}

\section{Introduction}

The hydroazulene framework is present in many natural products that are often associated with interesting biological properties [1,2]. The guaiane sesquiterpene (-)-oxyphyllol (1) has been isolated from the roots of the Thai medicinal plant Phyllanthus oxyphyllus [3]. A recent enantioselective synthesis of the unnatural enantiomer of $\mathbf{1}$ enabled a structural revision of this compound and established its relative and absolute configuration as depicted in Figure 1 [4]. During the total synthesis of (-)-9-deoxyenglerin A, compound $\mathbf{1}$ had already been prepared enantiomerically pure in 14 steps starting from (-)-isopulegol [5]. Remarkably, its cinnamate [(-)-9-deoxyenglerin A] displayed a cytotoxic activity in the $\mu \mathrm{M}$ range against several cancer cell lines [5]. Herein we report a concise enantioselective access to (-)-oxyphyllol (1) in a few preparatively simple operations.

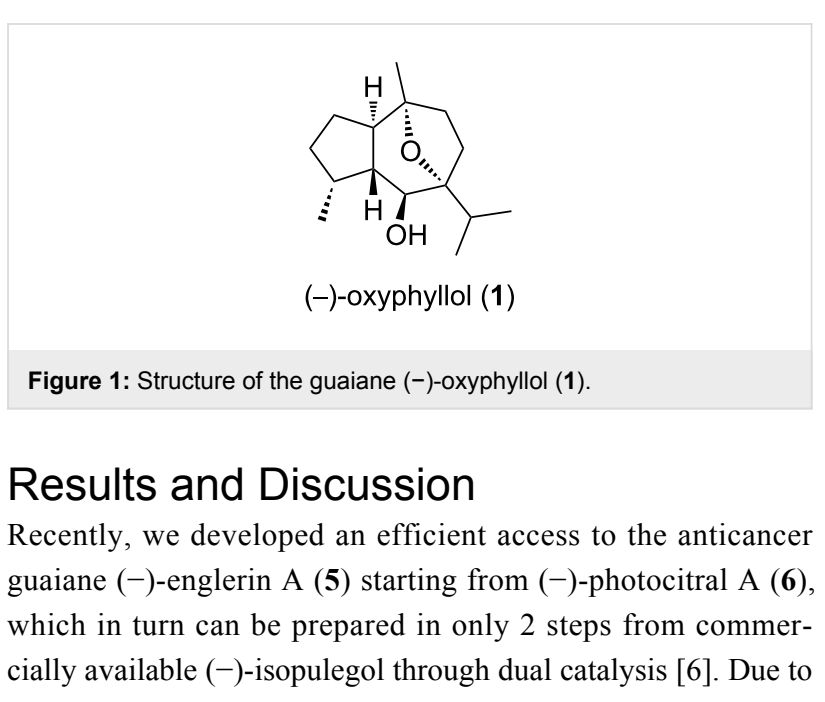


the structural similarity of the hydroazulene core in $(-)$-oxyphyllol (1) and $\mathbf{5}$, we decided to use a modification of our route to $\mathbf{5}$ for the synthesis of $\mathbf{1}$ that would also constitute a formal synthesis of the related guaiane (+)-orientalol E (2) $[4,7]$. As illustrated in Scheme 1, we selected tertiary alcohol 3 as a retrosynthetic precursor for $\mathbf{1}$. The acetyl group of $\mathbf{3}$ can be utilized to generate the isopropyl group, and a regioselective transannular epoxide opening would construct the oxygenbridged bicyclic hydroazulene framework. Alcohol 3 was traced back to the known epoxy enone 4 that already served as an intermediate for the total synthesis of 5 [6].

As shown in Scheme 2, we first planned to use the known diol 7, which is available by diastereoselective dihydroxylation of $\mathbf{4}$ [6]. Since a chemoselective deoxygenation of the secondary alcohol of 7 would give rise to the desired intermediate 3 , we investigated a radical defunctionalization strategy [8]. To this end, we tried to prepare the thionocarbonate 8 from 7 with phenyl chlorothionoformate $[9,10]$. However, all attempts at selective activation of the secondary hydroxy group led to the cyclic product 9 instead. A similar problem was already encountered earlier [11].

Scheme 3 depicts the efficacious completion of the synthesis of (-)-oxyphyllol (1) from 4. To our delight, a direct regio- and diastereoselective $\mathrm{Co}$ (II)-catalyzed hydration [12] of the olefin in 4 succeeded to give the required $\alpha$-stereoisomer 3 in $58 \%$ isolated yield after chromatographic separation of the minor $\beta$-alcohol. Compared to the envisaged deoxygenation route (Scheme 2), this key transformation saved 2 steps and paved the

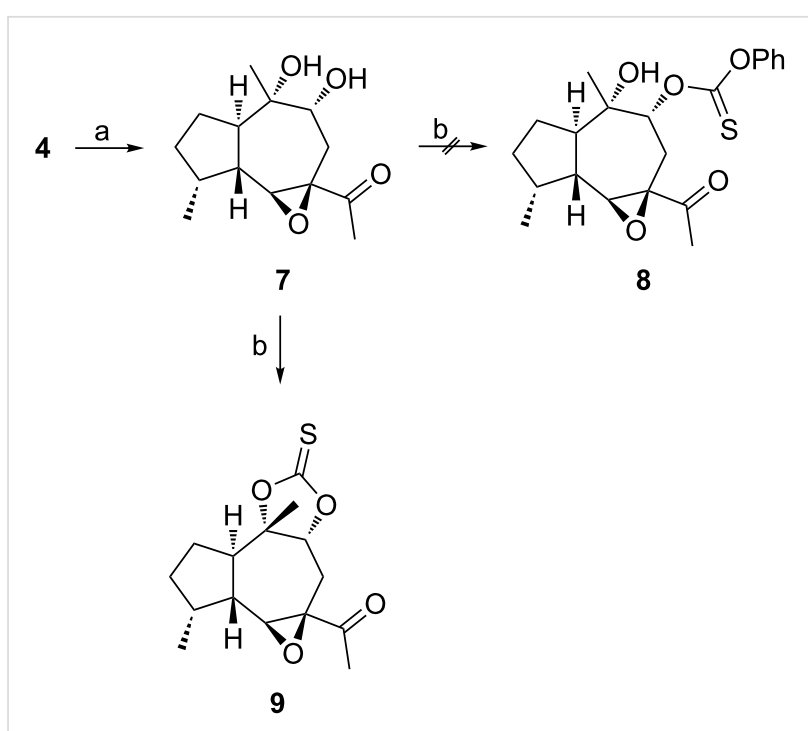

Scheme 2: Attempted selective deoxygenation of diol 7. a) $1 \mathrm{~mol} \%$ $\mathrm{K}_{2} \mathrm{OsO}_{4}, \mathrm{NMO}$, acetone, water, THF, rt, $97 \%$, diastereomeric ratio = 2:1 (ref. [6]); b) $\mathrm{PhOC}(\mathrm{S}) \mathrm{Cl}$, pyridine, $\mathrm{CH}_{2} \mathrm{Cl}_{2}, 0{ }^{\circ} \mathrm{C}$ to rt, $42 \% 9$. $\mathrm{NMO}=N$-methylmorpholine $N$-oxide.

way for a final reaction sequence that was based on our synthesis of (-)-englerin A (5) [6]. Thus, Wittig olefination of the acetyl group in $\mathbf{3}$ afforded the sensitive vinyl epoxide $\mathbf{1 0}$ along with some cyclized product $\mathbf{1 1}$. In contrast to the smooth transannular epoxide opening $[13,14]$ encountered during the synthesis of $\mathbf{5}$, an attempted complete cyclization of $\mathbf{1 0}$ to give 11 during acidic work-up of the methylenation reaction led to considerable decomposition. Fortunately, catalytic amounts of ytterbium triflate accomplished a high yielding formation of the

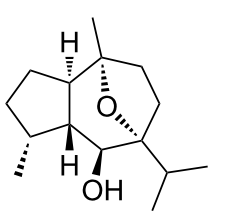

1

3 steps
(ref. [4])

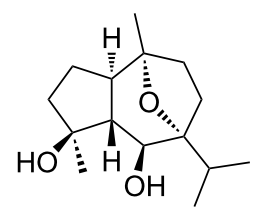

(+)-orientalol E (2)

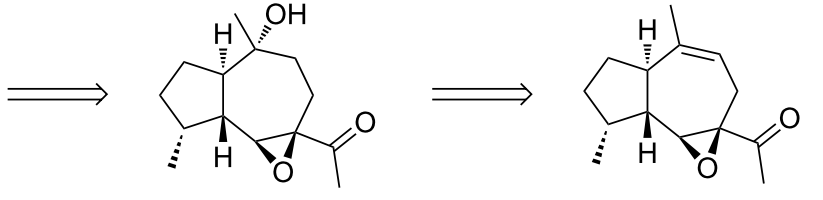

3

4
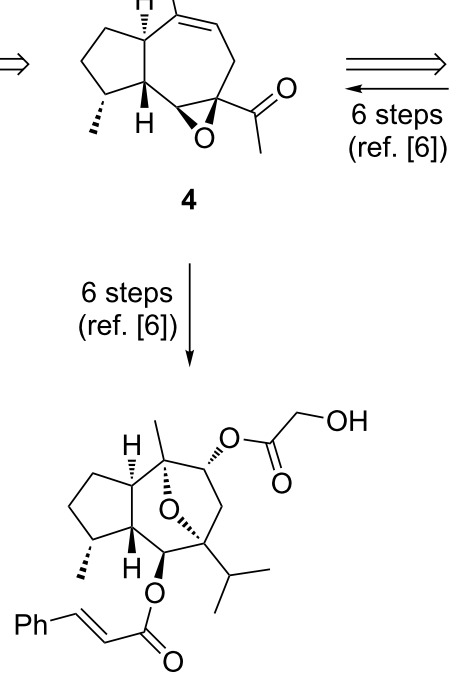

(-)-englerin A (5) 


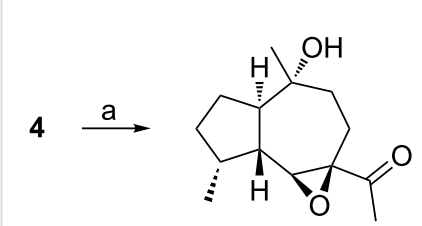

3

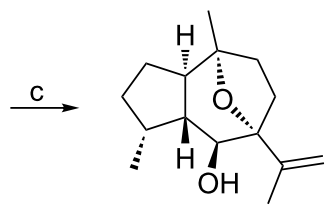

11
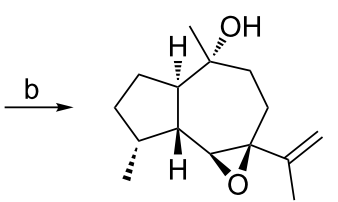

10
Scheme 3: Conversion of 4 to 1. a) $20 \mathrm{~mol} \% \mathrm{Co}(\mathrm{acac})_{2}, \mathrm{PhSiH}_{3}$, $1 \mathrm{~atm} \mathrm{O}_{2}$, THF, $0^{\circ} \mathrm{C}, 82 \%$, diastereomeric ratio $\left.=2.4: 1 ; \mathrm{b}\right) \mathrm{Ph}_{3} \mathrm{P}=\mathrm{CH}_{2}$, THF, rt; c) 2 mol \% Yb(OTf) $3, \mathrm{CH}_{2} \mathrm{Cl}_{2}, \mathrm{rt}, 80 \%$ (2 steps); d) $1 \mathrm{~atm} \mathrm{H}_{2}$, $10 \% \mathrm{Pd} / \mathrm{C}, \mathrm{MeOH}, \mathrm{rt}, 98 \%$. acac $=$ acetylacetonate, $\mathrm{Tf}=$ trifluoromethanesulfonyl.

oxygen-bridged bicyclic hydroazulene 11. Finally, hydrogenation of $\mathbf{1 1}$ proceeded uneventfully to deliver the target molecule $\mathbf{1}$ nearly quantitatively.

\section{Conclusion}

In summary, we have accomplished a short enantioselective total synthesis of (-)-oxyphyllol (1) and thus, a formal synthesis of (+)-orientalol E (2) as well by a modification of our previously developed route for setting up the oxygen-bridged framework present in (-)-englerin A (5). The reaction sequence presented herein allows the preparation of the guaiane $\mathbf{1}$ in only 10 steps with an overall yield of $22 \%$ starting from (-)-photocitral A (6).

\section{Experimental}

General information: Tetrahydrofuran and dichloromethane were dried and purified by passage through a MB-SPS-800 device using molecular sieves. All commercially available reagents were used as received. Reactions were performed under argon atmosphere. Thin layer chromatography (TLC) was performed on Merck silica gel $60 \mathrm{~F}_{254} 0.2 \mathrm{~mm}$ precoated plates. Product spots were visualized by UV light at $254 \mathrm{~nm}$ and subsequently developed using anisaldehyde solution as appropriate. Flash column chromatography was carried out using silica gel (Merck, particle size 40-63 microns). Melting points were measured on a Wagner \& Munz PolyTherm A and are uncorrected. Infrared spectra were recorded on a THERMONICOLET Avatar 360 instrument using ATR. NMR spectra were recorded on a Bruker DRX500P $\left(500.13 \mathrm{MHz}{ }^{1} \mathrm{H}, 125.77\right.$ $\left.\mathrm{MHz}{ }^{13} \mathrm{C}\right)$ or else on an Avance-III-600 $\left(600.16 \mathrm{MHz}{ }^{1} \mathrm{H}\right.$, $\left.150.92 \mathrm{MHz}{ }^{13} \mathrm{C}\right)$ spectrometer. Chemical shifts $(\delta)$ are quoted in parts per million ( $\mathrm{ppm}$ ) downfield of tetramethylsilane, using residual proton-containing solvent as internal standard $\left(\mathrm{CDCl}_{3}\right.$ at 7.27). Abbreviations used in the description of resonances are: s (singlet), d (doublet), $\mathrm{t}$ (triplet), q quartet), br (broad). Coupling constants $(J)$ are quoted to the nearest $0.1 \mathrm{~Hz}$. Mass spectra were recorded with an Agilent 5973N detector coupled with an Agilent $6890 \mathrm{~N}$ GC (GC-MS, $70 \mathrm{eV}$ ). HRMS spectra were recorded on a Finnigan MAT 95 (EI, 70 eV). Optical rotations were measured on a Perkin Elmer 341 LC polarimeter. Elemental analysis was performed on a Hekatech EA 3000.

Thionocarbonate 9: Diol 7 (10.0 mg, $39 \mu \mathrm{mol})$ and pyridine (6.2 mg, $78 \mu \mathrm{mol})$ were dissolved in dichloromethane $(0.5 \mathrm{~mL})$. The solution was cooled to $0{ }^{\circ} \mathrm{C}$, and phenyl chlorothionoformate $(10.3 \mathrm{mg}, 60 \mu \mathrm{mol})$ was added. The solution was warmed to room temperature and stirred for $72 \mathrm{~h}$. Then silica gel was added, and the solvents were removed in vacuo. Purification by flash chromatography (isohexane/ethyl acetate 2:1) afforded thionocarbonate 9 (4.9 $\mathrm{mg}, 17 \mu \mathrm{mol}, 42 \%)$ as a colorless solid.

9: $[\alpha]_{\mathrm{D}}{ }^{20}=-58\left(c 0.50, \mathrm{CHCl}_{3}\right) ; \mathrm{Mp}=203{ }^{\circ} \mathrm{C} ;{ }^{1} \mathrm{H} \mathrm{NMR}(600$ $\left.\mathrm{MHz}, \mathrm{CDCl}_{3}\right) \delta 4.62(\mathrm{dd}, J=11.7,3.8 \mathrm{~Hz}, 1 \mathrm{H}), 3.20(\mathrm{~s}, 1 \mathrm{H})$, $2.82(\mathrm{dd}, J=15.8,11.7 \mathrm{~Hz}, 1 \mathrm{H}), 2.63(\mathrm{dd}, J=15.6,3.6 \mathrm{~Hz}$, $1 \mathrm{H}), 2.54(\mathrm{dt}, J=12.1,8.8 \mathrm{~Hz}, 1 \mathrm{H}), 2.48-2.43(\mathrm{~m}, 1 \mathrm{H}), 2.19$ (dd, $J=12.4,7.2 \mathrm{~Hz}, 1 \mathrm{H}), 2.04(\mathrm{~s}, 3 \mathrm{H}), 2.03-1.88(\mathrm{~m}, 2 \mathrm{H})$, $1.65-1.58(\mathrm{~m}, 1 \mathrm{H}), 1.46(\mathrm{~s}, 3 \mathrm{H}), 1.43-1.37(\mathrm{~m}, 1 \mathrm{H}), 0.93(\mathrm{~d}, J=$ $7.2 \mathrm{~Hz}, 3 \mathrm{H}) \mathrm{ppm} ;{ }^{13} \mathrm{C} \mathrm{NMR}\left(150 \mathrm{MHz}, \mathrm{CDCl}_{3}\right) \delta 205.9(\mathrm{C})$, $190.4(\mathrm{C}), 91.6(\mathrm{C}), 85.5(\mathrm{CH}), 64.3(\mathrm{C}), 58.4(\mathrm{CH}), 44.5(\mathrm{CH})$, $41.1(\mathrm{CH}), 35.8(\mathrm{CH}), 31.2\left(\mathrm{CH}_{2}\right), 27.4\left(\mathrm{CH}_{2}\right), 24.9\left(\mathrm{CH}_{2}\right), 23.0$ $\left(\mathrm{CH}_{3}\right), 19.4\left(\mathrm{CH}_{3}\right), 16.9\left(\mathrm{CH}_{3}\right) \mathrm{ppm}$; IR (ATR) v: 2962, 2869 , 1789, 1701, 1291, 1253, 1152, $977 \mathrm{~cm}^{-1}$; GC-MS: $m / z=296$

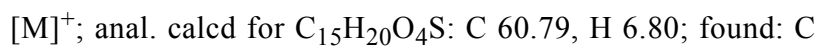
60.88, H 6.92 .

Alcohol 3: Epoxide $4(100.0 \mathrm{mg}, 454 \mu \mathrm{mol})$ and cobalt(II) acetylacetonate $(23.3 \mathrm{mg}, 91 \mu \mathrm{mol})$ were dissolved in THF $(5 \mathrm{~mL})$, and the solution was cooled to $0{ }^{\circ} \mathrm{C}$. Oxygen was bubbled through the solution for $1 \mathrm{~h}$, and phenylsilane (196.5 mg, $1.816 \mathrm{mmol}$ ) was added afterwards. After a reaction time of $22 \mathrm{~h}$, ethyl acetate was added, and the mixture was filtered through silica gel. The filtrate was concentrated under reduced pressure, and the residue was purified by flash chromatography (isohexane/ethyl acetate $2: 1$ ) to yield alcohol 3 (63.2 $\mathrm{mg}, 265 \mu \mathrm{mol}, 58 \%)$ as a colorless solid.

3: $[\alpha]_{\mathrm{D}}{ }^{20}=+3\left(c 0.54, \mathrm{CHCl}_{3}\right) ; \mathrm{Mp}=84{ }^{\circ} \mathrm{C} ;{ }^{1} \mathrm{H}$ NMR $(600$ $\left.\mathrm{MHz}, \mathrm{CDCl}_{3}\right) \delta 3.23(\mathrm{~d}, J=7.2 \mathrm{~Hz}, 1 \mathrm{H}), 2.55(\mathrm{ddd}, J=14.8$, 7.4, $1.5 \mathrm{~Hz}, 1 \mathrm{H}), 2.40-2.32(\mathrm{~m}, 1 \mathrm{H}), 2.03(\mathrm{~s}, 3 \mathrm{H}), 1.96-1.89$ $(\mathrm{m}, 1 \mathrm{H}), 1.85-1.80(\mathrm{~m}, 2 \mathrm{H}), 1.76-1.68(\mathrm{~m}, 2 \mathrm{H}), 1.64-1.51(\mathrm{~m}$, $2 \mathrm{H}), 1.38-1.31(\mathrm{~m}, 1 \mathrm{H}), 1.24-1.19(\mathrm{~m}, 1 \mathrm{H}), 1.13(\mathrm{~s}, 3 \mathrm{H}), 1.11$ $(\mathrm{d}, J=7.2 \mathrm{~Hz}, 3 \mathrm{H}) \mathrm{ppm} ;{ }^{13} \mathrm{C} \mathrm{NMR}\left(150 \mathrm{MHz}, \mathrm{CDCl}_{3}\right) \delta 208.2$ (C), $74.8(\mathrm{C}), 64.0(\mathrm{C}), 60.0(\mathrm{CH}), 52.1(\mathrm{CH}), 44.8(\mathrm{CH}), 40.8$ 
$\left(\mathrm{CH}_{2}\right), 35.9(\mathrm{CH}), 34.6\left(\mathrm{CH}_{2}\right), 25.4\left(\mathrm{CH}_{2}\right), 24.4\left(\mathrm{CH}_{2}\right), 23.3$ $\left(\mathrm{CH}_{3}\right), 19.9\left(\mathrm{CH}_{3}\right), 15.5\left(\mathrm{CH}_{3}\right) \mathrm{ppm}$; IR (ATR) v: 3446, 2957 , 2930, 2874, 1708, 1130, $852 \mathrm{~cm}^{-1}$; GC-MS: $m / z=238[\mathrm{M}]^{+}$; HRMS (EI) $m / z$ : $[\mathrm{M}]^{+}$calcd for $\mathrm{C}_{14} \mathrm{H}_{22} \mathrm{O}_{3}, 238.1569$; found, 238.1564 .

Allyl ether 11: Methyltriphenylphosphonium bromide (131.5 mg, $368 \mu \mathrm{mol})$ was suspended in THF $(1.5 \mathrm{~mL})$, and a solution of sodium hexamethyldisilazide in THF $(2.0 \mathrm{M}$, $172 \mu \mathrm{L}, 344 \mu \mathrm{mol})$ was added. The mixture was stirred for $5 \mathrm{~min}$ at room temperature, and then a solution of alcohol 3 $(29.0 \mathrm{mg}, 122 \mu \mathrm{mol})$ in THF $(1.5 \mathrm{~mL})$ was added dropwise After stirring for $5 \mathrm{~min}$ at room temperature, ethyl acetate was added, and the mixture was filtered through a plug of silica gel. Purification of the residue by flash chromatography (isohexane/ ethyl acetate 2:1) afforded a mixture of olefin $\mathbf{1 0}$ and the cyclized product $\mathbf{1 1}$ as a colorless oil.

The mixture above and ytterbium(III) triflate $(1.5 \mathrm{mg}, 2.4 \mu \mathrm{mol})$ were dissolved in dichloromethane $(1.5 \mathrm{~mL})$ and stirred for $1 \mathrm{~h}$ at room temperature. Afterwards the reaction mixture was filtered through silica gel, and the filtrate was evaporated in vacuo. Flash chromatography (isohexane/ethyl acetate 10:1) of the residue afforded the oxygen-bridged bicyclic hydroazulene 11 (22.9 mg, $97 \mu \mathrm{mol}, 80 \%$ over 2 steps) as a colorless oil.

11: $[\alpha]_{\mathrm{D}}{ }^{20}=-36(c 0.61, \mathrm{MeOH}) ;{ }^{1} \mathrm{H}$ NMR $\left(500 \mathrm{MHz}, \mathrm{CDCl}_{3}\right)$ $\delta 5.02(\mathrm{t}, J=1.6 \mathrm{~Hz}, 1 \mathrm{H}), 4.80(\mathrm{~s}, 1 \mathrm{H}), 3.38(\mathrm{~d}, J=10.4 \mathrm{~Hz}$, $1 \mathrm{H}), 2.30-1.38(\mathrm{~m}, 1 \mathrm{H}), 2.20-2.07(\mathrm{~m}, 1 \mathrm{H}), 2.06-1.92(\mathrm{~m}, 1 \mathrm{H})$, $1.86(\mathrm{~s}, 3 \mathrm{H}), 1.82-1.72(\mathrm{~m}, 2 \mathrm{H}), 1.71-1.60(\mathrm{~m}, 2 \mathrm{H}), 1.54-1.45$ $(\mathrm{m}, 1 \mathrm{H}), 1.41-1.36(\mathrm{~m}, 1 \mathrm{H}), 1.28(\mathrm{~s}, 3 \mathrm{H}), 1.22-1.13(\mathrm{~m}, 1 \mathrm{H})$, $1.10-1.00(\mathrm{~m}, 1 \mathrm{H}), 0.89(\mathrm{~d}, J=7.3 \mathrm{~Hz}, 3 \mathrm{H}) \mathrm{ppm} ;{ }^{13} \mathrm{C} \mathrm{NMR}$ $\left(125 \mathrm{MHz}, \mathrm{CDCl}_{3}\right) \delta 147.2(\mathrm{C}), 113.2\left(\mathrm{CH}_{2}\right), 88.0(\mathrm{C}), 82.9$ (C), $69.8(\mathrm{CH}), 48.9(\mathrm{CH}), 46.7(\mathrm{CH}), 31.4\left(\mathrm{CH}_{2}\right), 31.4\left(\mathrm{CH}_{2}\right)$, $31.1(\mathrm{CH}), 30.7\left(\mathrm{CH}_{2}\right), 26.0\left(\mathrm{CH}_{2}\right), 24.3\left(\mathrm{CH}_{3}\right), 19.3\left(\mathrm{CH}_{3}\right)$, $17.1\left(\mathrm{CH}_{3}\right) \mathrm{ppm}$; IR (ATR) v: 3467, 3082, 1073, 2952, 2869, $890 \mathrm{~cm}^{-1}$; GC-MS: $m / z=236[\mathrm{M}]^{+}$. HRMS (EI) $m / z:[\mathrm{M}]^{+}$ calcd for $\mathrm{C}_{15} \mathrm{H}_{24} \mathrm{O}_{2}, 236.1776$; found, 236.1766.

(-)-Oxyphyllol (1): Olefin $11(22.9 \mathrm{mg}, 97 \mu \mathrm{mol})$ and palladium on charcoal $(10 \mathrm{wt} \%, 5.1 \mathrm{mg})$ were placed in a flask, and methanol $(2 \mathrm{~mL})$ was added. The resulting mixture was stirred under hydrogen $(1 \mathrm{~atm})$ for $4 \mathrm{~h}$ at room temperature. Then the mixture was filtered through silica gel, and the solvents were removed in vacuo. Flash chromatography of the residue (isohexane/ethyl acetate 10:1) afforded $\mathbf{1}$ as a colorless oil (22.7 mg, $95 \mu \mathrm{mol}, 98 \%$ ).

1: $[\alpha]_{\mathrm{D}}{ }^{20}=-51\left(c 0.82, \mathrm{CHCl}_{3}\right)$; (Lit. [3]: $[\alpha]_{\mathrm{D}}{ }^{26}=-40.25(c$ $\left.\left.0.225, \mathrm{CHCl}_{3}\right)\right) ;{ }^{1} \mathrm{H}$ NMR $\left(500 \mathrm{MHz}, \mathrm{CDCl}_{3}\right) \delta 3.69(\mathrm{~d}, J=$ $10.1 \mathrm{~Hz}, 1 \mathrm{H}), 2.37-2.21(\mathrm{~m}, 1 \mathrm{H}), 2.05-1.87(\mathrm{~m}, 2 \mathrm{H}), 1.85-1.76$ (m, 1H), 1.75-1.54 (m, 4H), 1.44-1.32 (m, 2H), $1.23(\mathrm{~s}, 3 \mathrm{H})$, $1.21-1.13(\mathrm{~m}, 1 \mathrm{H}), 1.05-0.99(\mathrm{~m}, 1 \mathrm{H}), 1.03$ (d, $J=6.9 \mathrm{~Hz}, 3 \mathrm{H})$, $1.02(\mathrm{~d}, J=6.6 \mathrm{~Hz}, 3 \mathrm{H}), 0.88(\mathrm{~d}, J=7.3 \mathrm{~Hz}, 3 \mathrm{H}) \mathrm{ppm}$; ${ }^{13} \mathrm{C}$ NMR $\left(125 \mathrm{MHz}, \mathrm{CDCl}_{3}\right) \delta 86.4(\mathrm{C}), 83.0(\mathrm{C}), 71.8(\mathrm{CH})$, $49.3(\mathrm{CH}), 48.6(\mathrm{CH}), 32.4(\mathrm{CH}), 31.7\left(\mathrm{CH}_{2}\right), 31.5\left(\mathrm{CH}_{2}\right), 30.5$ (CH), $29.2\left(\mathrm{CH}_{2}\right), 25.9\left(\mathrm{CH}_{2}\right), 24.3\left(\mathrm{CH}_{3}\right), 18.4\left(\mathrm{CH}_{3}\right), 17.5$ $\left(\mathrm{CH}_{2}\right), 17.1\left(\mathrm{CH}_{3}\right) \mathrm{ppm}$; IR (ATR) v: 3408, 2953, 2870, 1473, 1378, 1064, $1004 \mathrm{~cm}^{-1}$; GC-MS: $m / z=238[\mathrm{M}]^{+}$.

\section{Supporting Information}

The Supporting Information contains ${ }^{1} \mathrm{H}$ and ${ }^{13} \mathrm{C}$ NMR spectra for compounds $\mathbf{1}, \mathbf{3 , 9}$ and 11 .

\section{Supporting Information File 1}

${ }^{1} \mathrm{H}$ NMR and ${ }^{13} \mathrm{C}$ NMR spectra.

[http://www.beilstein-journals.org/bjoc/content/ supplementary/1860-5397-9-239-S1.pdf]

\section{References}

1. Fraga, B. M. Nat. Prod. Rep. 2012, 29, 1334-1366. doi:10.1039/c2np20074k

2. Foley, D. A.; Maguire, A. R. Tetrahedron 2010, 66, 1131-1175. doi:10.1016/j.tet.2009.11.045

3. Sutthivaiyakit, S.; Nakorn, N. N.; Kraus, W.; Sutthivaiyakit, P. Tetrahedron 2003, 59, 9991-9995. doi:10.1016/j.tet.2003.10.023

4. Wang, J.; Chen, S.-G.; Sun, B.-F.; Lin, G.-Q.; Shang, Y.-J. Chem.-Eur. J. 2013, 19, 2539-2547. doi:10.1002/chem.201203467

5. Ushakov, D. B.; Navickas, V.; Ströbele, M.; Maichle-Mössmer, C.; Sasse, F.; Maier, M. E. Org. Lett. 2011, 13, 2090-2093. doi:10.1021/ol200499t

6. Zahel, M.; Keßberg, A.; Metz, P. Angew. Chem. 2013, 125, 5500-5502. doi:10.1002/ange.201301247 Angew. Chem., Int. Ed. 2013, 52, 5390-5392. doi:10.1002/anie.201301247

7. Peng, G.-P.; Tian, G.; Huang, X.-F.; Lou, F.-C. Phytochemistry 2003, 63, 877-881. doi:10.1016/S0031-9422(03)00222-X

8. Hartwig, W. Tetrahedron 1983, 39, 2609-2645. doi:10.1016/S0040-4020(01)91972-6

9. Robins, M. J.; Wilson, J. S. J. Am. Chem. Soc. 1981, 103, 932-933. doi:10.1021/ja00394a033

10. Wang, Y.; Metz, P. Chem.-Eur. J. 2011, 17, 3335-3337. doi:10.1002/chem.201003399

11. Jung, P. M. J.; Burger, A.; Biellmann, J.-F. J. Org. Chem. 1997, 62, 8309-8314. doi:10.1021/jo9704568

12. Isayama, S.; Mukaiyama, T. Chem. Lett. 1989, 18, 1071-1074. doi:10.1246/cl.1989.1071

13. Willot, M.; Radtke, L.; Könning, D.; Fröhlich, R.; Gessner, V. H.; Strohmann, C.; Christmann, M. Angew. Chem. 2009, 121, 9269-9272. doi:10.1002/ange.200905032 Angew. Chem., Int. Ed. 2009, 48, 9105-9108. doi:10.1002/anie.200905032

14. Yoshikawa, M.; Fukuda, Y.; Hatakeyama, S.; Tanaka, N.; Matsuda, H.; Yamahara, J.; Murakami, N. Chem. Pharm. Bull. 1993, 41, 1194-1196. doi:10.1248/cpb.41.1194 


\section{License and Terms}

This is an Open Access article under the terms of the Creative Commons Attribution License

(http://creativecommons.org/licenses/by/2.0), which permits unrestricted use, distribution, and reproduction in any medium, provided the original work is properly cited.

The license is subject to the Beilstein Journal of Organic Chemistry terms and conditions:

(http://www.beilstein-journals.org/bjoc)

The definitive version of this article is the electronic one which can be found at:

doi:10.3762/bjoc.9.239 